Thin-Layer Chromatographic $R_{F}$ Values of Toxicologically Relevant Substances on Standardized Systems

VCH Verlagsgesellschaft, Weinheim; 1987.223 pages with 2 figures and 15 tables. Price DM 68.-.

This volume of TLC RF data was published under the auspices of the Deutsche Forschungsgemeinschaft (DFG) and the International Association of Forensic Toxicologists (TIAFT). It was produced in response to the perceived need for compilation of reliable analytical data to aid in the identification of substances often used as poisons. The result is a set of $h R_{F}\left(R_{F} \times 100\right)$ information on some 1100 compounds (mostly pharmaceuticals and pesticides) in up to 10. standard solvent systems on silica gel TLC plates. As such it complements earlier publications of GC-retention indices.

The logic behind this approach, and some hints as to how to use the system, are given in a commendably brief introduction, whilst the bulk of text is given over to providing the $\mathrm{h}_{\mathrm{F}}$ data in tabular form. Indeed these tables take up some 196 pages out of a total of about 220. The results are provided in a variety of ways, either alphabetically by compound, or in ascending $h R_{F}$ value by solvent system. Each table for a particular solvent system also gives the $h R_{F}$ values for all the other solvent systems. Thus, for example on finding that, in solvent system 3 , the unknown has a h $R_{F}$ of 51 either ethoxyzolamide or adinazolam would be indicated. By rapidly scanning across the table it is clear that these possibilities could easily be distinguished by rerunning the sample in solvent systems 1 or 2 where they have quite different $R_{F}$ values.

This is clearly a very useful, logical and readily implemented approach, which would be even more so if the hRF data was complete for each compound in each system. In fact the data is rather patchy with some compounds covered fully and others described by a single entry (e.g. butylaminobenzoate and pramiverinel. Other compounds have multiple entries, but in all the systems described the hRF value is $O$ (e.g. pethidine intermediate $C$, cromoglicic acid); whilst it may be argued that these compounds are rather obscure it does come as a surprise to find that paraquat features only in 2 systems, and in both it has an $h R_{F}$ of $O$ ! This lack of information poses two prob. lems. Firstly identification of these compounds by such systems will be difficult, and secondly, it is unclear whether their chromatography in systems, for which information is not provided, will cause possible interference with the detection of any of the other compounds for which the hRF data is available.

Despite these limitations, which will hopefully be rectified in later editions, this is a very useful compilation of data. Indeed although aimed primarily at forensic toxicologists it should be useful to a wide range of chromatographers who wish to separate drugs by TLC.

1. D. Wilson

\title{
Errata
}

\section{Indirect Detection in Liquid Chromatography. Part I. Response Models for Reversed-Phase Ion- Pairing Systems}

\section{J.Crommen/G. Schill/D.Westerlund/L. Hackzell Chromatographia 24, 252-260 (1987)}

By mistake, Fig. 3 of the above paper has been reproduced incompletely. The correct figure is shown below.

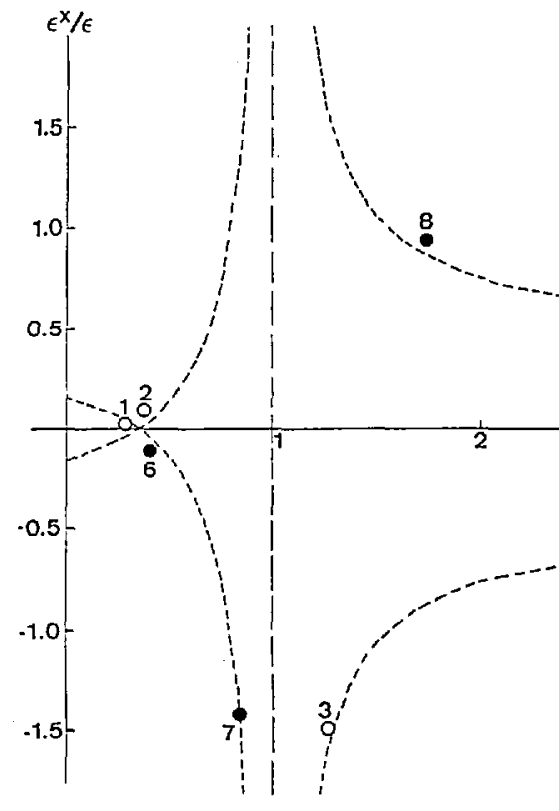

\section{An Efficient Procedure for Extraction and Determination of Steroids in the Tissue of Laboratory Animals}

\author{
A. Laganà / L. Curini/R. Curini/G.d'Ascenzo/A. Marino \\ Chromatographia 23, 796-802 (1987)
}

In the original publication of this paper, the names of the two authors Letizia Curini and Roberta Curini erroneously have been omitted. The authors' line should read as shown above. 